\title{
Optical and magneto-optical properties of Fe nanoparticles
}

\author{
J. L. Menéndez,* B. Bescós, and G. Armelles \\ Instituto de Microelectrónica de Madrid-IMM (CSIC), Isaac Newton 8 (PTM), Tres Cantos, 28760 Madrid, Spain
}

R. Serna and J. Gonzalo

Instituto de Optica, CSIC, Serrano 121, 28006 Madrid, Spain

R. Doole and A. K. Petford-Long

Department of Materials, University of Oxford, Parks Road, Oxford OX1 3PH, United Kingdom

\section{I. Alonso}

Institut de Ciència de Materials de Barcelona (CSIC), Campus Universitat Autònoma de Barcelona, 08193 Bellaterra, Spain

(Received 11 October 2001; revised manuscript received 31 January 2002; published 13 May 2002)

\begin{abstract}
The optical and magneto-optical properties of Fe nanoparticles with sizes ranging from 2 to $8 \mathrm{~nm}$, embedded in amorphous $\mathrm{Al}_{2} \mathrm{O}_{3}$, are studied as a function of their size and shape. The optical properties were measured using spectroscopic ellipsometry, whereas the magneto-optical properties were determined in two different Kerr configurations: polar and transverse. A generalization of different effective medium approximations is used to describe and analyze experimental data in nanocomposite media. In this generalization, the shapes of the nanoparticles are considered as an input parameter. The optical and magneto-optical parameters show clearly different values as a function of the nanoparticle size. A reasonable agreement between the theoretical calculations and experimental data is found when the average size of the nanoparticles is larger than $4 \mathrm{~nm}$. On the other hand, the experimental and theoretical curves differ for smaller sizes, implying that below $4 \mathrm{~nm}$ the optical and magneto-optical constants of the particles deviate from the bulk behavior.
\end{abstract}

DOI: 10.1103/PhysRevB.65.205413

\section{INTRODUCTION}

There has been a strong effort to investigate the properties of ferromagnetic nanoparticles in recent years. Most of these works were devoted to studying the magnetic interaction between the particles ${ }^{1-3}$ and the modifications of the magnetic properties as a function of size. ${ }^{4,5}$ In most of these works the sizes of the particles were limited to $5-10 \mathrm{~nm}$, a size range within which the intrinsic magnetic properties of the particles are already similar to those of bulk material. Despite this interest, the number of studies of the optical and magneto-optical properties of ferromagnetic nanoparticles is scarce. $^{6-8}$ Nevertheless, the optical and magneto-optical properties are known to be very sensitive to the electronic structure of the materials, and, therefore, they should reflect the changes in the electronic structure of the nanoparticles, if they take place as the size is reduced. In this work the optical and magneto-optical properties of Fe nanoparticles with sizes in the range of 2-8 nm, embedded in an $\mathrm{Al}_{2} \mathrm{O}_{3}$ matrix, are studied as a function of size and taking into account their shapes. A generalization of an effective-medium description of the dielectric tensor of nanoparticles embedded in a matrix has been made to treat actual samples where the particles have different shapes and are randomly oriented. Different configurations of the applied magnetic field are also considered. A change in the optical and magneto-optical properties of the particles is observed as the particle size is reduced.

\section{EXPERIMENT}

Three nominally 18-nm $\quad \mathrm{Al}_{2} \mathrm{O}_{3} / 5\left[\left(2.5 \mathrm{~nm} \mathrm{Fe}: \mathrm{Al}_{2} \mathrm{O}_{3}\right) /\right.$ $\left.18-\mathrm{nm} \mathrm{Al}_{2} \mathrm{O}_{3}\right] / \mathrm{Si}$ films were grown by pulsed laser deposi-
PACS number(s): 61.46.+w, 78.20.Ls, 78.66.Vs, 81.15.Fg

tion using an ArF excimer laser beam $(\lambda=193 \mathrm{~nm}, \tau$ $=20 \mathrm{~ns}$ full width at half maximum). The laser beam was sequentially focused on the surface of high-purity $\mathrm{Al}_{2} \mathrm{O}_{3}$ and Fe rotating targets with an average energy density of $3 \mathrm{~J} / \mathrm{cm}^{2}$. The films were grown in vacuum $\left(1 \times 10^{-6} \mathrm{mbar}\right)$ on Si substrates held at room temperature, and placed $32 \mathrm{~mm}$ away from the target surface. The number of pulses on the $\mathrm{Fe}$ target was varied in order to change the Fe content. The samples produced that way will be referred to from now on as samples $A, B$, and $C$, sample $A(C)$ being the one with the smallest (largest) Fe content. Further details of the synthesis procedure of the nanocomposite films can be found elsewhere. ${ }^{9}$ The samples were characterized by highresolution transmission electron microscopy (HRTEM) using a film with a sandwich structure of $\mathrm{Al}_{2} \mathrm{O}_{3} /(\mathrm{Fe}$ nanoparticles in $\left.\mathrm{Al}_{2} \mathrm{O}_{3}\right) / \mathrm{Al}_{2} \mathrm{O}_{3}$ deposited on carbon-coated mica substrates grown under equivalent conditions to the multilayers. This was done in order to prevent the overlap of images from the different layers with $\mathrm{Fe}$ particles in the transmission electron microscopy analysis. The period and thickness of the different multilayers were obtained by low-angle x-ray reflectometry (XRR).

The optical properties of the layers containing nanoparticles were studied in the spectral range $1-4.5 \mathrm{eV}$ using a spectroscopic ellipsometer with a rotating polarizer. The measured ellipsometric spectra were interpreted using a multilayer model. The parameters in such a model are the thickness and dielectric function of each layer, in addition to the known sequence of layers forming the structures. To reduce the number of unknowns we have fixed the layer thicknesses to those measured by XRR, and the refractive index of $\mathrm{Al}_{2} \mathrm{O}_{3}$ to a reference value previously determined. ${ }^{10}$ Thus 

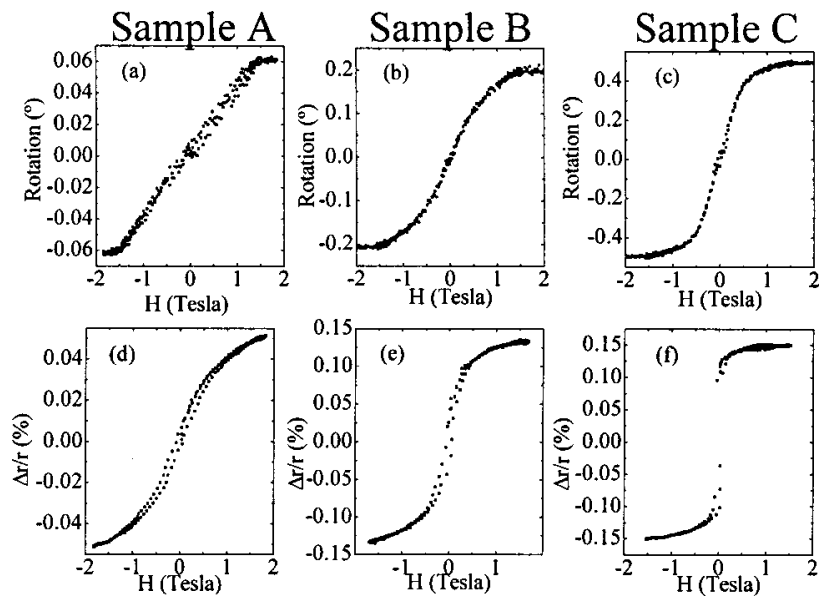

FIG. 1. Kerr loops in the polar [(a)-(c)] and transverse [(d)-(f)] configurations for samples $A, B$ and $C$, respectively.

the only unknowns were the optical functions $n$ and $k$ of the layers containing the Fe nanoparticles. Both spectra, measured at $60^{\circ}$ and $70^{\circ}$ of incidence, were fitted simultaneously to extract the optical properties of these layers.

The magneto-optical characterization was carried out in the polar and transverse Kerr configurations in the spectral range $1.5-4.5 \mathrm{eV}$. In the polar configuration the magnetic field is applied perpendicular to the plane of the sample (along the $z$ direction) and the angle of incidence of the light is $5^{\circ}$, the applied magnetic field being of $1.5 \mathrm{~T}$, enough to saturate the samples, as shown in Figs. 1(a)-1(c). The experimentally determined parameters in this configuration are the Kerr rotation and ellipticity.

In the transverse configuration the magnetic field is applied in the plane of the sample (along the $x$ direction) and perpendicular to the plane of incidence ( $y z$ plane). The applied magnetic field was $0.15 \mathrm{~T}$, which is not enough to reach magnetic saturation in these samples, as shown in Figs. 1(d) $-1(\mathrm{f})$. The transverse Kerr data were corrected for saturation using Kerr loops at a fixed wavelength. In this configuration the parameter measured is the variation of the reflectivity of light polarized in the plane of incidence, due to the applied magnetic field, normalized to the reflectivity at zero field. The transverse spectra were taken at two angles of incidence in each sample: $40^{\circ}$ and $70^{\circ}$.

\section{RESULTS}

Figure 2 shows HRTEM planar view pictures of the three samples: $A$ [Fig. 2(a)], $B$ [Fig. 2(b)], and $C$ [Fig. 2(c)]. The
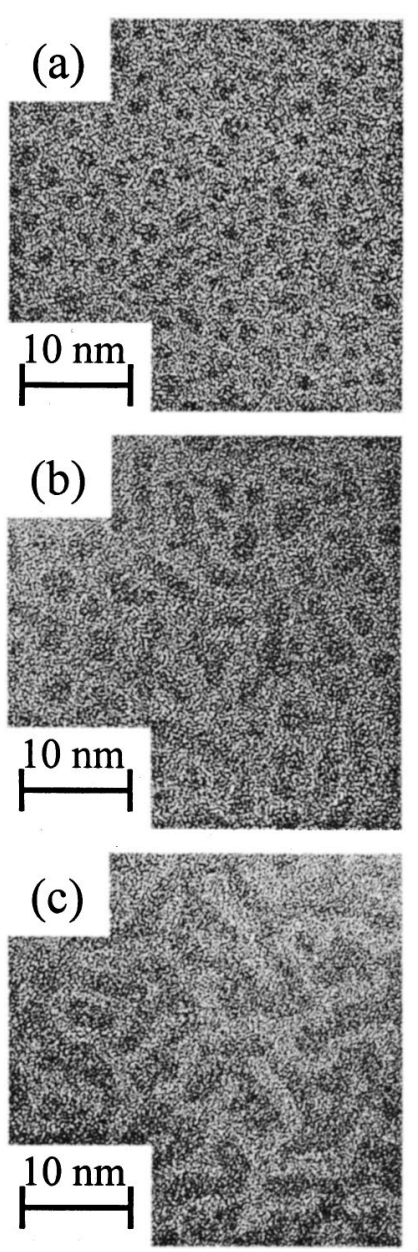
$C$ (c)

FIG. 2. TEM images corresponding to samples $A,(\mathrm{a}), B$ (b), and

particles in sample A, Fig. 2(a) have a nearly circular inplane shape with a rather uniform distribution. As the amount of $\mathrm{Fe}$ is increased, sample $B$, some of the particles coalesce, leading to more elongated or cylindrical shapes; with their size also increasing [Fig. 2(b)]. In sample $C$, the coalescence is very important, leading to ramified clusters [Fig. 2(c)]. However, the formalism developed in the Appendix considers ellipsoidal particles. The approximation made, in order to apply the theory, considered each of the branches of the clusters as an individual ellipsoid. The validity of this approximation will be discussed below. The mean values of length and breadth for the different samples obtained from these images are given in Table I.

TABLE I. Lengths, breadths, and heights; shape factors $L x, L y$, and $L z$ for the perturbative solution and the local Fe concentration for the different samples.

\begin{tabular}{cccccccc}
\hline \hline Sample & $\begin{array}{c}\text { Length } \\
(\mathrm{nm})\end{array}$ & $\begin{array}{c}\text { Breadth } \\
(\mathrm{nm})\end{array}$ & $\begin{array}{c}\text { Height } \\
(\mathrm{nm})\end{array}$ & $\begin{array}{c}\text { Fe } \\
\text { concentration } \\
(\%)\end{array}$ & $L x$ & $L y$ & $L z$ \\
\hline$A$ & 2.4 & 1.6 & 2.5 & 10 & 0.3006 & 0.4831 & 0.2163 \\
$B$ & 4 & 2.6 & 2.7 & 30 & 0.229 & 0.3898 & 0.3812 \\
$C$ & 8 & 2 & 2.7 & 40 & 0.0905 & 0.5276 & 0.3819 \\
\hline \hline
\end{tabular}




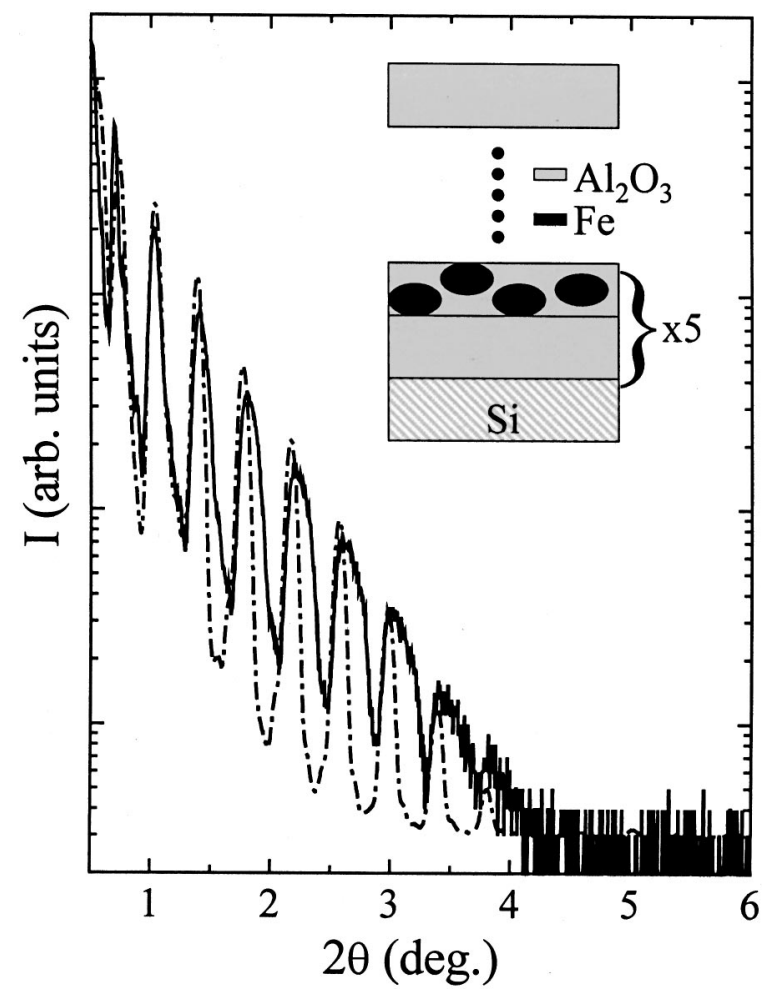

FIG. 3. XRR data (continuous line) and corresponding simulation (dot-dashed line) for sample $C$. The inset shows a layout of the multilayers structure.

XRR measurements were performed in order to determine the thickness of each individual layer in the different samples. As an example, Fig. 3 shows the scan obtained for the sample $C$ together with a layout of the actual structure of the samples. The solid line corresponds to the experimental data, while the dot-dashed line corresponds to a simulation of the experimental results. The difference between the experimental data and the simulation, mainly the broadening of the experimental scan vs. the simulation, is due to inhomogeneities in the multilayer thickness. The average thickness of the nanoparticles layers obtained with XRR is very similar to the size of the particles perpendicular to the growth plane obtained from grazing-incidence small-angle $\mathrm{x}$-ray scattering measurements, ${ }^{11}$ and ranges between 1.5 and $3 \mathrm{~nm}$. Therefore, the thickness obtained by XRR can also be identified with the height of the islands. The thickness of the pure $\mathrm{Al}_{2} \mathrm{O}_{3}$ layer and the nanoparticle layer are shown in Table II.

The local volume fraction of the particles in the layer containing nanoparticles can be obtained using the TEM re-

TABLE II. Thickness of the pure $\mathrm{Al}_{2} \mathrm{O}_{3}$ and the nanoparticle layers.

\begin{tabular}{ccc}
\hline \hline Sample & $\begin{array}{c}\mathrm{Al}_{2} \mathrm{O}_{3} \text { thickness } \\
(\mathrm{nm})\end{array}$ & $\begin{array}{c}\text { Nanoparticle layer } \\
\text { thickness }(\mathrm{nm})\end{array}$ \\
\hline$A$ & 17 & 2.5 \\
$B$ & 18 & 2.7 \\
$C$ & 18.5 & 2.7 \\
\hline \hline
\end{tabular}

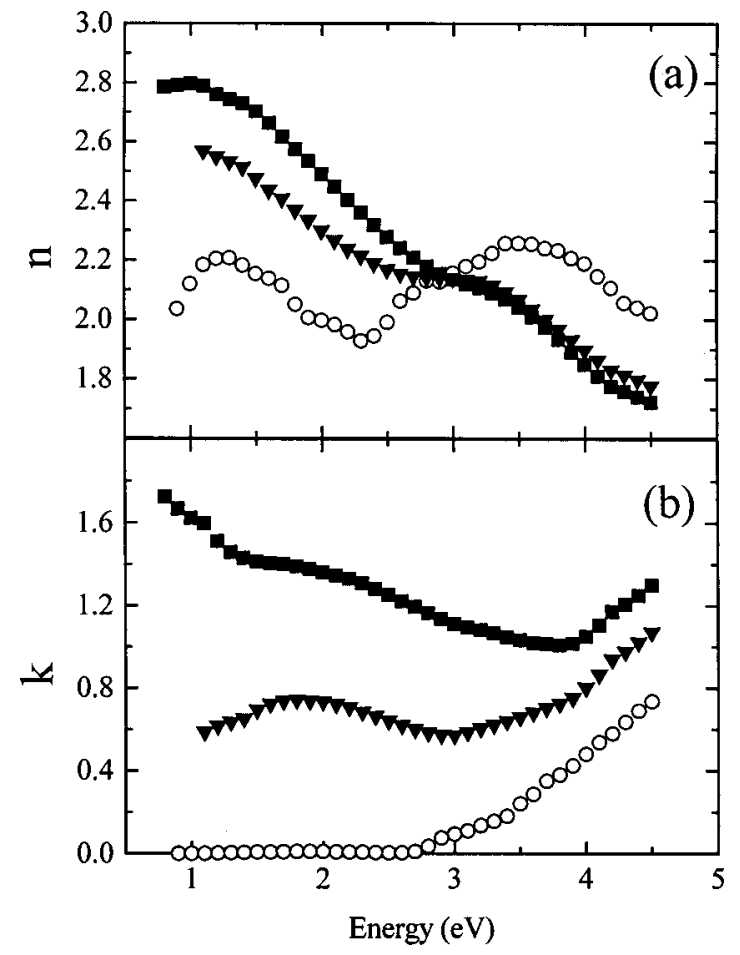

FIG. 4. Measured real $n$ (a) and imaginary $k$ (b) parts of the refractive indices corresponding to the composite layers in samples $A$ (open circles), $B$ (full triangles), and $C$ (full squares).

sults and the perpendicular size of the particles, and it varies between $10 \%$ and $40 \%$ for the different samples. In addition, an independent estimation of the concentration of $\mathrm{Fe}$ in the nanoparticles layer can also be made from the XRR data. As the electronic density of the nanoparticles layer is intermediate between that of pure $\mathrm{Al}_{2} \mathrm{O}_{3}$ and that of pure $\mathrm{Fe}$, an interpolation between these two values allows one to estimate the Fe concentration, the results obtained being consistent with those from the above-mentioned method. This way, the estimated Fe concentration in the nanocomposite layer was $10 \%$, $30 \%$, and $40 \%$ for samples $A, B$, and $C$, respectively. These values are also shown in Table I.

The index of refraction $n$ and the extinction coefficient $k$ of the nanoparticles layer for the different samples as obtained from the ellipsometry analysis are given in Fig. 4. The real part of the refractive index, $n$ [Fig. 4(a)], is similar for samples $B$ and $C$, decreasing its value for higher energies, while sample $A$ shows a flatter behavior. On the other hand, the imaginary part of the refractive index, $k$ [Fig. 4(b)], shows a decrease when the concentration of $\mathrm{Fe}$ in the layer is reduced.

Figure 5 shows both the Kerr ellipticity [Fig. 5(a)] and rotation [Fig. 5(b)] for the three characterized samples. The most relevant features in the spectra are the peaks around 4-4.5 eV for both the rotation and the ellipticity in all the samples that are due to interference effects. As expected, the positions where the interference maxima occur are mainly dependent on the individual thickness of the layers and, as these are very similar in the different samples, the maxima occur at similar positions in energy. 


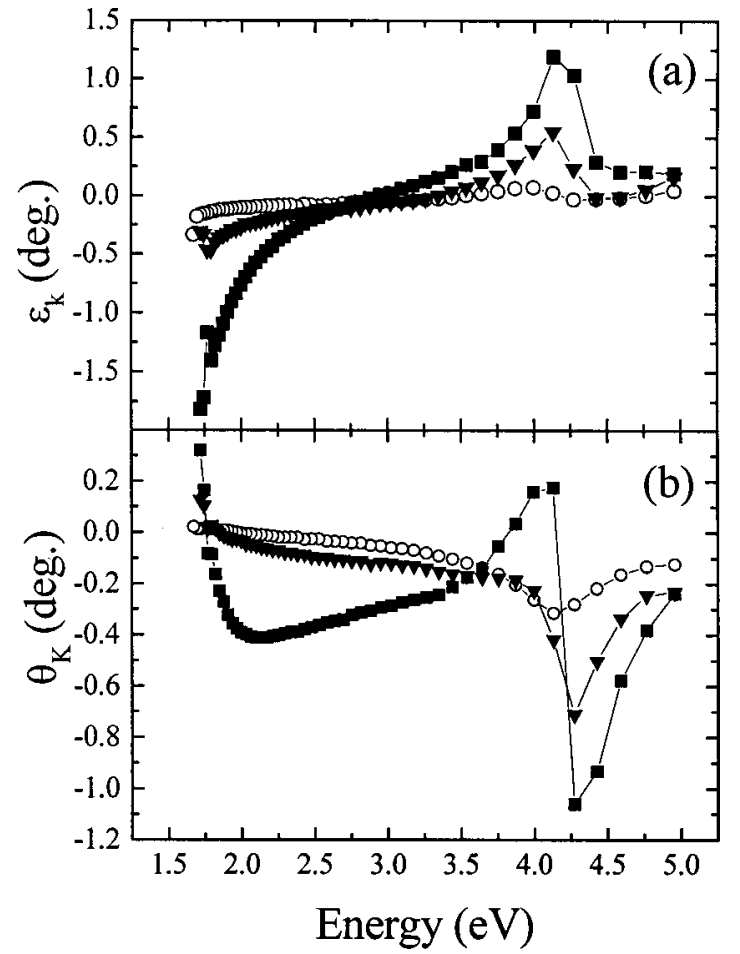

FIG. 5. Polar Kerr ellipticity (a) and rotation (b) for the three different samples: $A$ (open circles), $B$ (full triangles), and $C$ (full squares).

The transverse Kerr spectra of the samples measured at $40^{\circ}$ and $70^{\circ}$ of incidence are shown in Fig. 6. As in the preceding figure, the most relevant features in the spectra are the peaks around $1.8 \mathrm{eV}$ for the $40^{\circ}$ spectrum and $3 \mathrm{eV}$ for the $70^{\circ}$ spectrum, which are due to interference effects. In order to demonstrate that those peaks are due to interference effects, Fig. 7 shows how a simulation considering the bulk optical and magneto-optical constants of $\mathrm{Fe}$ and amorphous $\mathrm{Al}_{2} \mathrm{O}_{3}$ reproduces both the position and intensity of the peaks around 1.7 and $1.9 \mathrm{eV}$ (continuous line). However, if the thickness of the pure $\mathrm{Al}_{2} \mathrm{O}_{3}$ layers are changed to $16 \mathrm{~nm}$, the position of these peaks changes, therefore showing that their origin are interferences.

The transfer matrix formalism ${ }^{12}$ has been used in order to obtain the nondiagonal elements of the dielectric tensor. The only unknown parameters of the structure, which are fitted from the experimental data, are the real and imaginary parts of $\varepsilon_{x y}$ of the nanoparticles layer in the polar configuration and the real and imaginary parts of the ratio $i \varepsilon_{y z} / \varepsilon_{z z}$ of the nanoparticle layers for the transverse configuration. The thicknesses of the layers were those obtained in the XRR analysis. On the other hand, the refractive indices are those obtained in the analysis of the ellipsometry data and shown in Fig. 4. The experimental results obtained this way together with the theoretical calculations are shown in Figs. 8, 9, and 10 for samples $A, B$, and $C$, respectively. The most remarkable features in these figures is that, for every dielectric tensor element, the match between the experimental and theoretical curves is closer when the sizes of the particles studied are larger.

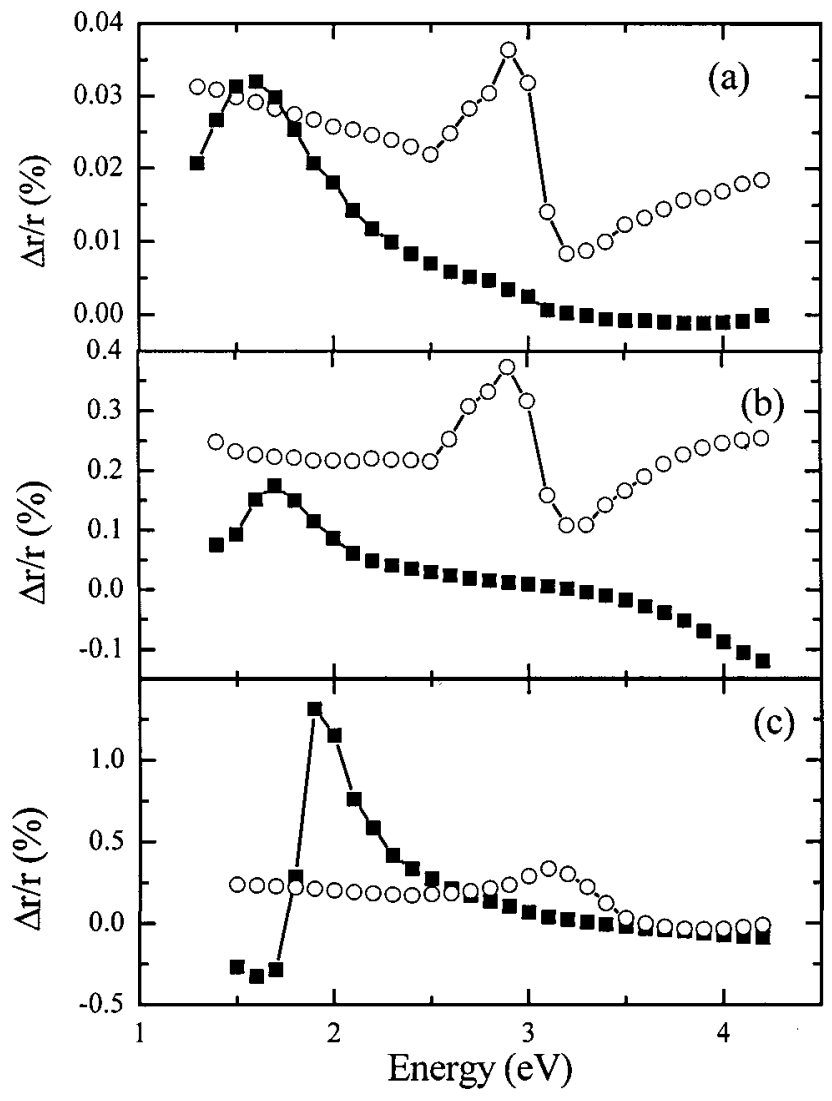

FIG. 6. Transverse Kerr spectra of samples $A(\mathrm{a}), B(\mathrm{~b})$, and $C$ (c) measured at two different angles of incidence, $40^{\circ}$ (full squares) and $70^{\circ}$ (open circles).

\section{DISCUSSION}

In the analysis of the optical and magneto-optical data previously shown, it has been assumed that the layers containing nanoparticles can be treated as layers having an effective dielectric tensor. This is a good approximation because of the small size of the particles in the three samples when compared to the wavelengths used in the experiments. This effective dielectric tensor depends on the dielectric tensor of the particles and the matrix and on the shape of the particles. In the Appendix, the expressions used in this work for the different elements of the effective dielectric tensor are derived for both polar and transverse configurations. The system is considered to consist of particles with ellipsoidal shapes with two of their principal axes in the film plane but randomly oriented, which corresponds to the actual structure of the samples. In order to calculate the elements of the dielectric tensor theoretically, several parameters must be used. The elements of the dielectric tensor used were those of bulk $\mathrm{Fe}$ (Ref. 13) and amorphous $\mathrm{Al}_{2} \mathrm{O}_{3} \cdot{ }^{10}$ The shape factors, $L_{i}$, were calculated according to Ref. 14 (see Table I) from the mean values of lengths and breadths and heights indicated above. As a first approach, in order to simplify the analysis, it has been considered that all the particles in the different samples have the same shape, that is, ellipsoids with equivalent $a / b$ and $a / c$ ratios, $a, b$, and $c$ being the ellipsoids axes. The value for the $\mathrm{Fe}$ concentration in the effective layers was that obtained from the XRR measure- 


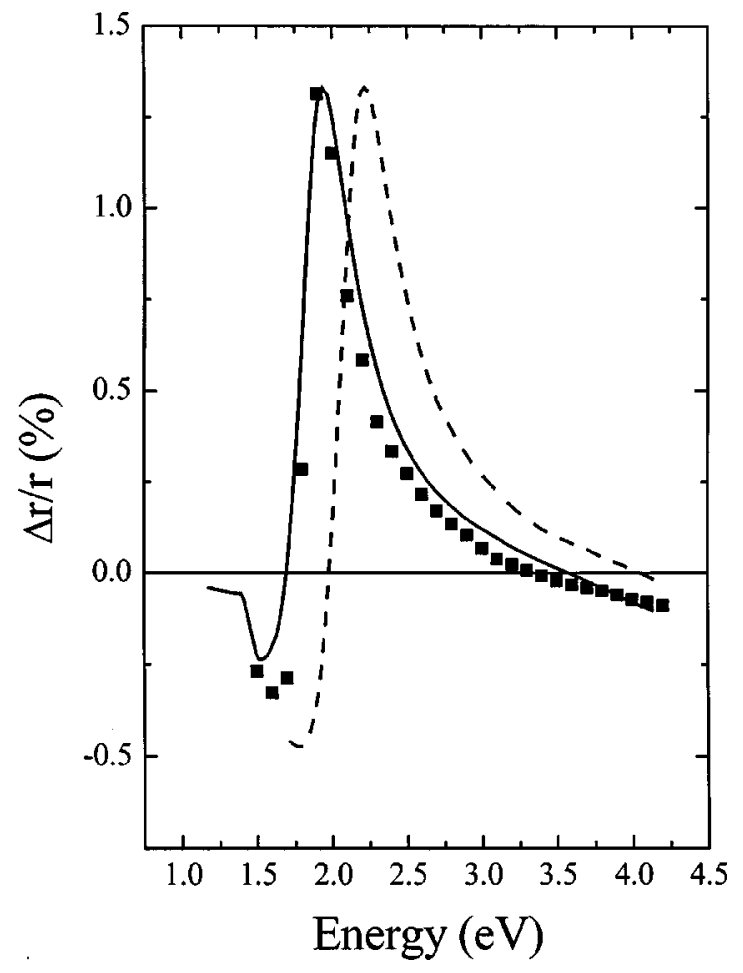

FIG. 7. Transverse Kerr spectra of sample $C$ at $40^{\circ}$ (full squares) and two simulations, one of them considering the experimentally determined thicknesses and bulk parameters (continuous line) and a second one (dotted line) where the $\mathrm{Al}_{2} \mathrm{O}_{3}$ thickness in the multilayer has been changed to $16 \mathrm{~nm}$ instead of $18.5 \mathrm{~nm}$.

ments and presented in Table I. With these values it is possible to calculate the diagonal and off-diagonal elements of the effective dielectric tensor according to formulas derived in the Appendix. The results are summarized here for the two approximations used, self-consistent and perturbative, respectively.

It is clear that none of the simulations follows the experimental curves for sample A [Figs. 8(a)-8(f)], for which the particles have average dimensions around $2 \mathrm{~nm}$. There is no match between the experiment and model for any of the components of the dielectric tensor, the difference being particularly noticeable for $\varepsilon_{x x}$. In the case of sample $B$ [Figs. 9(a)-9(f)], the agreement is better, especially regarding the spectral shape, although it is still far from being good. For sample $C$ [Figs. 10(a)-10(f)], the agreement of the experimental data with the self-consistent approach is much more reasonable than the perturbative approach, especially if only the experimental trends are considered, although it is still not satisfactory. In this point, it should be noted that the selfconsistent approach leads to theoretical curves that are much closer to the experimental results than those resulting from the perturbative solution. For low concentrations, both theories yield similar results as observed in the simulations performed for sample $A$.

There are several possibilities that could account for the difference between the experimental and theoretical results. First, the magnetic particles in the samples have been modeled as all having the same shape; this approach is true for the sample A, but not for the other samples. To illustrate the

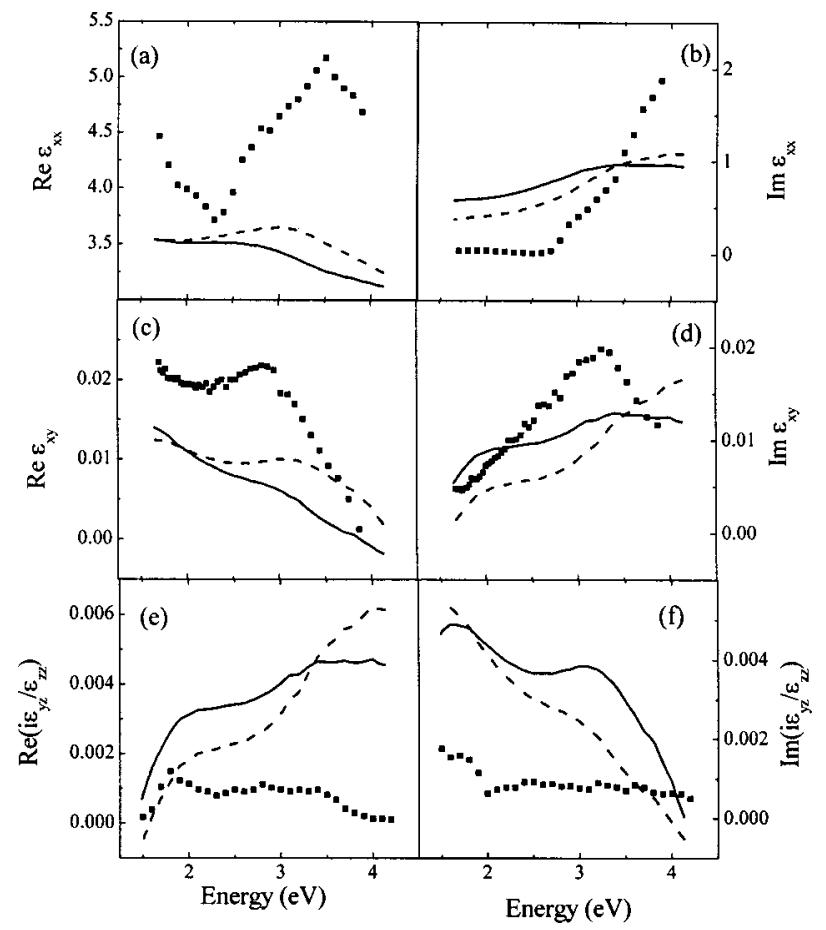

FIG. 8. Diagonal real (a) and imaginary (b) and nondiagonal real (c) and imaginary (d) parts of the dielectric tensor; and real (e) and imaginary (f) parts of the ratio $i \varepsilon_{y z} / \varepsilon_{z z}$ for sample $A$. Black squares account for the experimental data, while the continuous (dashed) line represents the self-consistent (perturbative) approach.

influence of particles having different shapes, simulations considering more than one shape have been performed under the self-consistent approach which is more accurate. However, in the present case, for the experimentally determined shapes, the difference between considering one or more shapes leads to very similar results and therefore, the presence of different shapes is not able to explain the spectral differences observed.

Up to this point, it has been considered that the alumina is pure, both in the $\mathrm{Al}_{2} \mathrm{O}_{3}$ and in the composite layers. However, the $\mathrm{Fe}$ atoms may be incorporated into the $\mathrm{Al}_{2} \mathrm{O}_{3}$ layer during deposition not only in the form of particles, but might also be dissolved throughout the film. The main effect of this incorporation of the Fe into the $\mathrm{Al}_{2} \mathrm{O}_{3}$ matrix as a dopant is an increase of the refractive index with respect to that of the pure $\mathrm{Al}_{2} \mathrm{O}_{3}$ matrix. The effect that such an increase has on the dielectric tensor is presented in the dotted lines of Figs. 10(a)-10(f) for sample $C$. In this case, the refractive index of the $\mathrm{Al}_{2} \mathrm{O}_{3}$ matrix, $n$, has been increased by 0.3 . As can be observed the agreement between theory and the experimental results is better, particularly in $\varepsilon_{x x}$, which points to the presence of dissolved $\mathrm{Fe}$ in the alumina layer. However, the agreement is not good even for this sample, indicating that other structural factors, such as possible inhomogeneities not considered in this analysis, are still present. It should be noted here that the approximation by which the clusters in sample $C$ were decomposed in individual ellipsoids is correct, as this is sample that shows a better agreement between theory and experiment.

Provided that the Fe nanoparticles are embedded in 


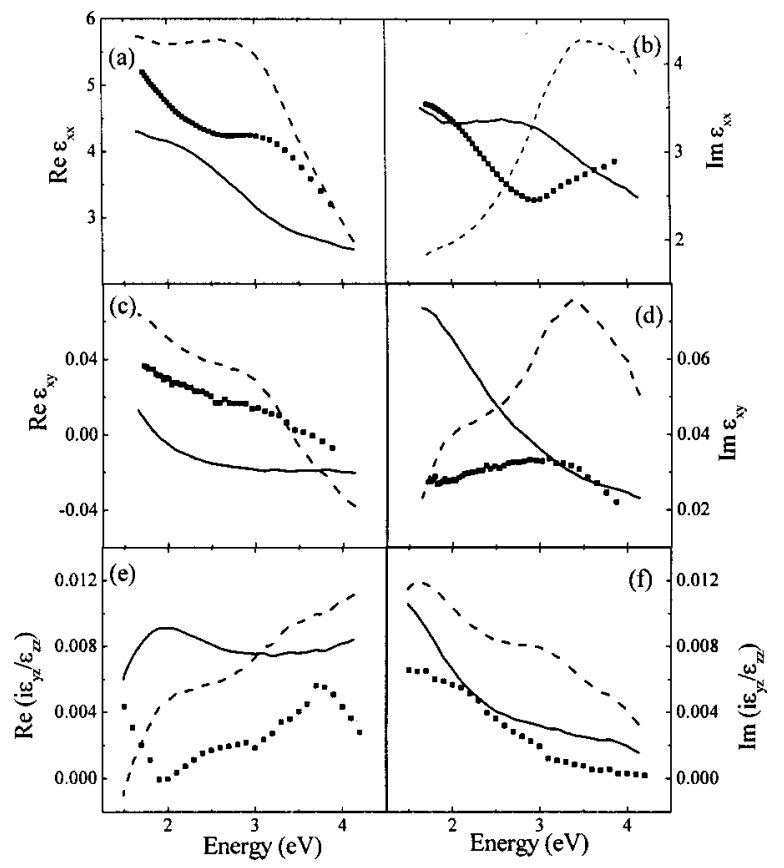

FIG. 9. Diagonal real (a) and imaginary (b) and nondiagonal real (c) and imaginary (d) parts of the dielectric tensor; and real (e) and imaginary (f) parts of the ratio $i \varepsilon_{y z} / \varepsilon_{z z}$ for sample $B$. Black squares account for the experimental data, while the continuous (dashed) line represents the self-consistent (perturbative) approach.

$\mathrm{Al}_{2} \mathrm{O}_{3}$, one must discuss the possibility of an Fe oxide shell being formed around the Fe nanoparticles. In Ref. 9 it was found, according to magnetization measurements, that the maximum thickness of an $\mathrm{Fe}$ shell formed around the $\mathrm{Fe}$ nanoparticles should be around $0.4 \mathrm{~nm}$, that is, around 2-3 atomic layers. In that work, it was established that the core of the Fe nanoparticles was $\alpha$-Fe and the shell a nonmagnetic Fe oxide. That estimation was performed under the hypothesis that there was no $\mathrm{Fe}$ dissolved in the $\mathrm{Al}_{2} \mathrm{O}_{3}$ matrix. However, as discussed above, there seems to be a certain amount of $\mathrm{Fe}$ dissolved in the $\mathrm{Al}_{2} \mathrm{O}_{3}$ matrix, therefore implying that the maximum thickness for the Fe oxide shell is under three atomic layers.

However, none of the possibilities already discussed improves the agreement between theory and experiment for sample $A$. On the other hand, for sample $B$, and even to a greater extent for sample $C$, there is a better agreement between theory and experiment, that is, the agreement improves for larger particle size. This suggests that the optical and magneto-optical properties of particles with sizes below 3-4 nm deviate from those of bulk Fe. This result agrees with the observed behavior of the enhanced magnetic moments in free-standing Fe particles. ${ }^{15}$ Also, modifications of the band structure have been reported in Cu particles ${ }^{16}$ and in the optical properties of $\mathrm{Cu} / \mathrm{Pt}$ particles ${ }^{17}$ of similar sizes to the Fe particles reported here.

The different elements of the dielectric tensor are not all affected in the same way: the nondiagonal elements suffer some changes induced by modifications in the electronic structure of the particles, but the most dramatic changes appear in the diagonal tensor elements. In particular, this can be

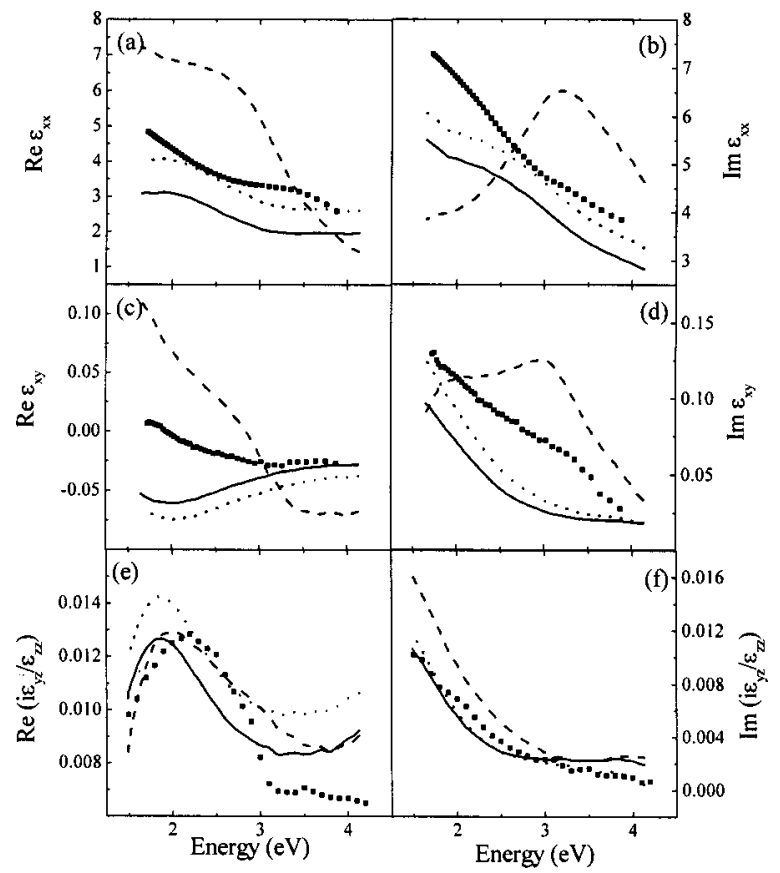

FIG. 10. Diagonal real (a) and imaginary (b) and nondiagonal real (c) and imaginary (d) parts of the dielectric tensor; and real (e) and imaginary (f) parts of the ratio $i \varepsilon_{y z} / \varepsilon_{z z}$ for sample $C$. Black squares account for the experimental data, while the continuous (dashed) line represents the self-consistent (perturbative) approach. The dotted line represents a simulation where the refractive index of amorphous $\mathrm{Al}_{2} \mathrm{O}_{3}$ has been increased by 0.3 .

observed for sample $A$, where the largest discrepancy between theory and experiment is in $\varepsilon_{x x}$. A similar observation was also reported in Ref. 18, where the influence of a distortion in $\mathrm{Fe}$ thin films was found to have a more noticeable effect on the diagonal elements of the conductivity tensor than on the nondiagonal elements.

Finally, it should be noted how the theoretical models developed in this work are able to reproduce electromagnetic resonances associated to the plasmons. For example, in sample $A$ [see Fig. 8(b)], the imaginary part of $\varepsilon_{x x}$ increases at higher energies, while in bulk Fe it decreases as it does in Fig. 10(b), where the Fe concentration in the nanoparticles layers is around $40 \%$. This increase is characteristic of plasmonlike resonances. It can be shown that if the matrix refractive index is increased, the peak associated with this plasmon resonance shifts to lower energies.

\section{CONCLUSIONS}

A set of multilayers with embedded Fe particles in the nanometer range has been grown and studied both structurally, optically and magneto-optically. The magneto-optical characterization was performed both in the polar and transverse configurations. In the different samples, the Fe concentration and the shapes and sizes of the nanoparticles have been varied. A theory, generalizing the classic MaxwellGarnett and Bruggeman approaches, has been derived in order to consider the shapes of the particles, and tested in these systems. 
The optical and magneto-optical activities of multilayer samples with the structure $18-\mathrm{nm} \quad \mathrm{Al}_{2} \mathrm{O}_{3} / 5$ $\times\left[\left(2.5-\mathrm{nm} \mathrm{Fe}: \mathrm{Al}_{2} \mathrm{O}_{3}\right) / 18-\mathrm{nm} \mathrm{Al}_{2} \mathrm{O}_{3}\right] / \mathrm{Si}$ have been studied, and the dielectric tensor of the nanocomposite layers $\left(\mathrm{Fe}: \mathrm{Al}_{2} \mathrm{O}_{3}\right)$ has been calculated theoretically and experimentally determined. For particle sizes larger than $4 \mathrm{~nm}$, the match between the theoretical calculations and the experimental curves is reasonable. However, below that size, the experimental and theoretical curves show larger discrepancies and for the smallest particles studied, around $2 \mathrm{~nm}$ in diameter, their optical and magneto-optical experimental and theoretical curves show the maximum discrepancies. This evolution strongly suggests that the optical and magnetooptical properties of nanometer size particles and, therefore, their electronic structure, deviate from the bulk behavior when their dimensions are below $4 \mathrm{~nm}$.

\section{ACKNOWLEDGMENTS}

J. L. M. wants to acknowledge Comunidad de Madrid's Consejería de Educación y Cultura for financial support. This work was partially supported by TIC99-0866, MAT98-0974, MAT99-1063, CICYT (Spain).

\section{APPENDIX}

The optical properties of a composite material having particles randomly distributed with dimensions much smaller that the wavelength of light can be described using an effective-medium approximation. In this, the optical properties of the material are described by an effective dielectric tensor $\varepsilon_{e}$, which depends on the dielectric functions of the matrix and particle material, concentration and shape of the particles through following relation ${ }^{19,20}$

$$
\varepsilon_{e}-\varepsilon_{0}=\left\langle(1-\delta \varepsilon \Gamma)^{-1}\right\rangle^{-1}\left\langle(1-\delta \varepsilon \Gamma)^{-1} \delta \varepsilon\right\rangle,
$$

where $\delta \varepsilon=\varepsilon(r)-\varepsilon_{0}, \varepsilon(r)$ is the dielectric function of the matrix or particle material, $\varepsilon_{0}$ is an arbitrarily defined reference dielectric tensor, the brackets denote a volume average, and $\Gamma$ is a tensor which depends on the shape of the particles. The choice of $\varepsilon_{0}$ determines the approximation used. Two cases will be considered. In the first one, $\varepsilon_{0}$ is taken equal to $\varepsilon_{e}$, a self-consistent solution known as Bruggeman approximation, whereby Eq. (A1) is then reduced to

$$
\left\langle(1-\delta \varepsilon \Gamma)^{-1} \delta \varepsilon\right\rangle=0 .
$$

In the second case $\varepsilon_{0}$ is taken equal to the matrix dielectric tensor $\varepsilon_{m}$, a perturbative approach known as the MaxwellGarnett approximation.

Let us consider the case of a composite material made of a nonmagnetic matrix with an isotropic dielectric tensor $\varepsilon_{m}$ and magnetic particles embedded in it. The magnetic material has the following dielectric tensor if it is fully magnetized along the $x$ direction:

$$
\left(\begin{array}{ccc}
\varepsilon_{p} & 0 & 0 \\
0 & \varepsilon_{p} & i \delta_{p} \\
0 & -i \delta_{p} & \varepsilon_{p}
\end{array}\right),
$$

This is, for example, the case of Fe if the very small difference between the $x x$ and $y y$ components, proportional to the square of the magnetization, is neglected.

In an analogous way, when the magnetization is along the $z$ direction,

$$
\left(\begin{array}{ccc}
\varepsilon_{p} & -i \delta_{p} & 0 \\
i \delta_{p} & \varepsilon_{p} & 0 \\
0 & 0 & \varepsilon_{p}
\end{array}\right),
$$

It will be assumed that if the particles of the composite material are fully magnetized along the $x$ direction the effective dielectric tensor can be written as

$$
\left(\begin{array}{ccc}
\varepsilon_{e}^{x} & 0 & 0 \\
0 & \varepsilon_{e}^{y} & i \delta_{e} \\
0 & -i \delta_{e} & \varepsilon_{e}^{z}
\end{array}\right)
$$

Similarly, when the magnetization is along the $z$ direction the effective dielectric tensor takes the form

$$
\left(\begin{array}{ccc}
\varepsilon_{e}^{x} & -i \delta_{e} & 0 \\
i \delta_{e} & \varepsilon_{e}^{y} & 0 \\
0 & 0 & \varepsilon_{e}^{z}
\end{array}\right)
$$

The theory will be developed for particles with ellipsoidal shapes, with two of the three principal axes lying in the $x y$ plane and randomly oriented. In that case the expressions for the different components of the dielectric tensor can be easily obtained and will be given for the two solutions already discussed. Quadratic terms in $\delta$ will be neglected. In the following, the superscripts and subscripts $m$ and $i$ will refer to the matrix material and to the different shapes of the magnetic material(s), respectively. On the other hand, $f_{i}$ corresponds to the volumic fraction of particles with an ellipsoidal shape $i$.

\section{Self-consistent solution $\varepsilon_{0}=\varepsilon_{e}$}

For the case of the self-consistent solution, Eq. (A2) leads for the diagonal elements to

$$
\begin{gathered}
\left(1-\Sigma f_{i}\right)\left(\varepsilon_{m}-\varepsilon_{e}^{x}\right)\left\{1 /\left[1-\left(\varepsilon_{m}-\varepsilon_{e}^{x}\right) \Gamma_{x}^{m}\right]+\Sigma f_{i}\left(\varepsilon_{p}-\varepsilon_{e}^{x}\right) /\right. \\
2\left\{1 /\left[1-\left(\varepsilon_{p}-\varepsilon_{e}^{x}\right) \Gamma_{x}^{i}\right]+1 /\left[1-\left(\varepsilon_{p}-\varepsilon_{e}^{x}\right) \Gamma_{y}^{i}\right]\right\}=0 \\
\varepsilon_{e}^{x}=\varepsilon_{e}^{y} \\
\left(1-\Sigma f_{i}\right)\left(\varepsilon_{m}-\varepsilon_{e}^{z}\right)\left\{1 /\left[1-\left(\varepsilon_{m}-\varepsilon_{e}^{z}\right) \Gamma_{z}^{m}\right]\right. \\
+\Sigma f_{i}\left(\varepsilon_{p}-\varepsilon_{e}^{z}\right)\left\{1 /\left[1-\left(\varepsilon_{p}-\varepsilon_{e}^{z}\right) \Gamma_{z}^{i}\right]\right\}=0
\end{gathered}
$$

For the nondiagonal elements in the transverse configuration, 


$$
\begin{aligned}
(1- & \left.\Sigma f_{i}\right)\left(-\delta_{e}\right)\left\{1 /\left[1-\left(\varepsilon_{m}-\varepsilon_{e}^{y}\right) \Gamma_{x}^{m}\right]\right\}\left\{1 /\left[1-\left(\varepsilon_{m}-\varepsilon_{e}^{z}\right) \Gamma_{z}^{m}\right]\right\} \\
& +\Gamma f_{i}\left(\delta_{p}-\delta_{e}\right) / 2\left\{1 /\left[1-\left(\varepsilon_{p}-\varepsilon_{e}^{x}\right) \Gamma_{x}^{i}\right]\right. \\
& \left.+1 /\left[1-\left(\varepsilon_{p}-\varepsilon_{e}^{x}\right) \Gamma_{y}^{i}\right]\right\}\left\{1 /\left[1-\left(\varepsilon_{p}-\varepsilon_{e}^{z}\right) \Gamma_{z}^{i}\right]\right\}=0, \quad \text { (A10) }
\end{aligned}
$$

In the polar configuration,

$$
\begin{gathered}
\left(1-\Sigma f_{i}\right)\left(-\delta_{e}\right)\left\{1 /\left[1-\left(\varepsilon_{m}-\varepsilon_{e}^{x}\right) \Gamma_{x}^{m}\right]\right\}^{2}+\Sigma f_{i}\left(\delta_{p}-\delta_{e}\right) \\
\times\left\{1 /\left[1-\left(\varepsilon_{p}-\varepsilon_{e}^{x}\right) \Gamma_{x}^{i}\right]\right\}\left\{1 /\left[1-\left(\varepsilon_{p}-\varepsilon_{e}^{y}\right) \Gamma_{y}^{i}\right]\right\}=0 .
\end{gathered}
$$

$\Gamma_{j}^{i}=-L_{j}^{i} / \varepsilon_{e}^{j}$, with $L_{j}^{i}$ being the components of the depolarization tensor with respect to the principal axis of an ellipsoid having axis $\left(a_{j}^{i} / \sqrt{\varepsilon_{m}}\right)$, and $a_{j}$ is the dimension of the $j$ th principal axis of that particle.

The matrix is considered to be composed of spherical particles with radius $b$ and, therefore, $\Gamma_{j}^{m}=-L_{j} / \varepsilon_{e}^{j}$, with $L_{j}$ the $j$ th component of the depolarization tensor with respect to the principal axis of an ellipsoid having axis $\left(b / \sqrt{\varepsilon_{e}^{j}}\right)$.

\section{Perturbative solution $\varepsilon_{0}=\varepsilon_{m}$}

If $\varepsilon_{0}$ is taken equal to $\varepsilon_{m}$, which is known as the perturbative solution, and only the linear terms in $\delta$ are kept, the diagonal terms are equal to

$$
\varepsilon_{e}^{x}=\varepsilon_{e}^{y}=\varepsilon_{m}+A_{1}^{1} / A_{1}
$$

where the coefficients $A_{1}^{1}$ and $A_{1}$ are

$$
\begin{aligned}
A_{1}^{1}= & \left(\varepsilon_{p}-\varepsilon_{m}\right) \Gamma_{i}\left(f_{i} / 2\right)\left\{1 /\left[1-\left(\varepsilon_{p}-\varepsilon_{m}\right) \Gamma_{x}^{i}\right]\right. \\
& \left.+1 /\left[1-\left(\varepsilon_{p}-\varepsilon_{m}\right) \Gamma_{y}^{i}\right]\right\}, \\
A_{1}= & \left\{1+\left(\varepsilon_{p}-\varepsilon_{m}\right) \Sigma_{i}\left(f_{i} / 2\right)\left(\Gamma_{x}^{i} /\left[1-\left(\varepsilon_{p}-\varepsilon_{m}\right) \Gamma_{x}^{i}\right]\right.\right. \\
+ & \left.\Gamma_{y}^{i} /\left[1-\left(\varepsilon_{p}-\varepsilon_{m}\right) \Gamma_{y}^{i}\right]\right\},
\end{aligned}
$$

and

$$
\varepsilon_{e}^{z}=\varepsilon_{m}+A_{3}^{1} / A_{3}
$$

the coefficients $A_{3}^{1}$ and $A_{3}$ have the following expressions:

$$
\begin{gathered}
A_{3}^{1}=\left(\varepsilon_{p}-\varepsilon_{m}\right) \Sigma_{i} f_{i}\left\{1 /\left[1-\left(\varepsilon_{p}-\varepsilon_{m}\right) \Gamma_{z}^{i}\right]\right\}, \\
A_{3}=1+\left(\varepsilon_{p}-\varepsilon_{m}\right) \Sigma_{i} f_{i} \Gamma_{z}^{i} /\left[1-\left(\varepsilon_{p}-\varepsilon_{m}\right) \Gamma_{z}^{i}\right] .
\end{gathered}
$$

Regarding the nondiagonal terms, $\delta_{e}$ has the following expression in the transverse configuration:

$$
\delta_{e}=\delta_{p} B^{1} /\left(A_{1} A_{3}\right),
$$

the coefficient $B^{1}$ being

$$
\begin{aligned}
B^{1}= & \Sigma_{i}\left(f_{i} / 2\right)\left\{1 /\left[1-\left(\varepsilon_{p}-\varepsilon_{m}\right) \Gamma_{x}^{i}\right]+1 /\left[1-\left(\varepsilon_{p}-\varepsilon_{m}\right) \Gamma_{y}^{i}\right]\right\} \\
& \times\left\{1 /\left[1-\left(\varepsilon_{p}-\varepsilon_{m}\right) \Gamma_{z}^{i}\right]\right\}
\end{aligned}
$$

On the other hand, for the polar configuration

$$
\delta_{e}=\delta_{p}\left(C^{1}-C_{1}\right) / C,
$$

the coefficients $C_{1}, C^{1}$, and $C$ being

$$
\begin{aligned}
C_{1}= & \Sigma_{i}\left(f_{i} / 2\right)\left(\varepsilon_{p}-\varepsilon_{m}\right)\left(\Gamma_{x}^{i}+\Gamma_{y}^{i}\right)\left\{1 /\left[1-\left(\varepsilon_{p}-\varepsilon_{m}\right) \Gamma_{x}^{i}\right]\right\} \\
& \times\left\{1 /\left[1-\left(\varepsilon_{p}-\varepsilon_{m}\right) \Gamma_{y}^{i}\right]\right\} \\
C^{1}= & \Sigma_{i} f_{i}\left\{1 /\left[1-\left(\varepsilon_{p}-\varepsilon_{m}\right) \Gamma_{x}^{i}\right]\right\}\left\{1 /\left[1-\left(\varepsilon_{p}-\varepsilon_{m}\right) \Gamma_{y}^{i}\right]\right\} \\
C= & \left\{1+\Sigma\left(f_{i} / 2\right)\left(\varepsilon_{p}-\varepsilon_{m}\right)\left\{\Gamma_{x}^{i} /\left[1-\left(\varepsilon_{p}-\varepsilon_{m}\right) \Gamma_{x}^{i}\right]\right.\right. \\
& \left.+\Gamma_{y}^{i} /\left[1-\left(\varepsilon_{p}-\varepsilon_{m}\right) \Gamma_{y}^{i}\right]\right\}
\end{aligned}
$$

$\Gamma_{j}^{i}=-L_{j}^{i} / \varepsilon_{m}$, with $L_{j}^{i}$ being the components of the depolarization tensor with respect to the principal axis of an ellipsoid having axis $\left(a_{j}^{i} / \sqrt{\varepsilon_{m}}\right)$. The superscript $i$ refers to the $i$ th shape of the particles, and $a_{j}$ is the dimension of the $j$ th principal axis of that particle. It should be noted that the polarization tensor does not depend on the wavelength in the perturbative solution, but for nonspherical particles it does depend on the wavelength in the self-consistent approach.
*Corresponding author: J. L. Menéndez, Instituto de Microelectrónica de Madrid (CNM-CSIC), C/Isaac Newton, 8-PTM, 28760 Tres Cantos, Madrid (Spain). Telephone: 349180607 00; FAX: 349180607 01; Email address: menendez@imm.cnm.csic.es

${ }^{1}$ A. Hernando, J. Phys.: Condens. Matter 11, 9455 (1999).

${ }^{2}$ J. F. Löffler, H.-B. Benjamin Braun, and W. Wagner, Phys. Rev. Lett. 85, 1990 (2000).

${ }^{3}$ E. Bonetti, L. Del Bianco, D. Fiorani, D. Rinaldi, R. Caciuffo, and A. Hernando, Phys. Rev. Lett. 83, 2829 (1999).

${ }^{4}$ K. W. Edmonds, C. Binns, S. H. Baker, S. C. Thornton, C. Norris, J. B. Goedkoop, M. Finazzi, and N. Brookes, Phys. Rev. B 60, 472 (1999).

${ }^{5}$ J. L. Dormann, D. Fiorani, F. Giammaria, and F. Lucari, J. Appl. Phys. 69, 5130 (1991).

${ }^{6}$ Z. S. Jiang, G. J. Jin, J. T. Ji, H. Sang, Y. W. Du, S. M. Zhou, Y. D. Wang, and L. Y. Chen, J. Appl. Phys. 78, 439 (1995).
${ }^{7}$ V. G. Kravets, A. K. Petford-Long, and A. F. Kravets, J. Appl. Phys. 87, 1762 (2000).

${ }^{8}$ M. Abe and M. Gomi, Jpn. J. Appl. Phys. 23, 1580 (1984).

${ }^{9}$ N. M. Dempsey, L. Ranno, D. Givord, J. Gonzalo, R. Serna, G. T. Fei, A. K. Petford-Long, R. C. Doole, and D. E. Hole, J. Appl. Phys. 90, 6268 (2001).

${ }^{10}$ R. Serna, J. C. G. de Sande, J. M. Ballesteros, and C. N. Afonso, J. Appl. Phys. 84, 4509 (1998).

${ }^{11}$ A. Naudon (private communication).

${ }^{12}$ M. Schubert, Phys. Rev. B 53, 4265 (1996).

${ }^{13}$ J. H. Weaver, E. Colavita, D. W. Lynch, and R. Rosei, Phys. Rev. B 19, 3850 (1979).

${ }^{14}$ S. Berthier, J. Phys. I 4, 303 (1994).

${ }^{15}$ J. Shi, S. Gider, K. Babcock, and D. D. Awschalom, Science 271, 937 (1996).

${ }^{16}$ E. Anno, M. Tanimoto, and T. Yamaguchi, Phys. Rev. B 38, 3521 (1988). 
${ }^{17}$ K. Uchida, S. Kaneko, S. Omi, C. Hata, H. Tanji, Y. Asahara, A. J. Ikushima, T. Tokizaki, and A. Nakamura, J. Opt. Soc. Am. B 11, 1236 (1994).

${ }^{18}$ J. L. Menéndez, G. Armelles, A. Cebollada, D. Weller, and A.
Delin, Phys. Rev. B 62, 10498 (2000).

${ }^{19}$ D. Stroud, Phys. Rev. B 12, 3368 (1975).

${ }^{20}$ J. S. Ahn, K. H. Kim, T. W. Noh, D.-H. Riu, H.-K. Boo, and H.-E. Kim, Phys. Rev. B 52, 15244 (1995). 